\title{
Pressure-induced Lifshitz and structural transitions in NbAs and TaAs: Experiments and Theory
}

\author{
Satyendra Nath Gupta ${ }^{1}$, Anjali Singh ${ }^{2 \dagger}$, Koushik $\mathrm{Pal}^{2 \dagger}$, \\ D.V.S.Muthu ${ }^{1}$, C. Shekhar ${ }^{3}$, Moaz A. Elghazali ${ }^{3,4}$, Pavel G. \\ Naumov $^{3}$, Sergey A. Medvedev ${ }^{3}$, C. Felser ${ }^{3}$, U V Waghmare $^{2}$ \\ and A. K. Sood ${ }^{1,5}$ \\ $\ddagger$ \\ E-mail: asood@iisc.ac.in \\ ${ }^{1}$ Department of Physics, Indian Institute of Science, Bangalore-560012,India \\ ${ }^{2}$ Theoretical Sciences Unit, Jawaharlal Nehru Centre for Advanced Scientific \\ Research, Bangalore-560064,India ${ }^{3}$ Max Planck Institute for Chemical Physics of \\ Solids, 01187 Dresden, Germany ${ }^{4}$ Institute for Solid State Physics, Technical \\ University Dresden, 01069 Dresden, Germany ${ }^{5}$ International Centre for Materials \\ Science, Jawaharlal Nehru Centre for Advanced Scientific Research, Bangalore \\ 560064 , India ${ }^{\dagger}$ These two authors contributed equally
}

\begin{abstract}
High pressure Raman, resistivity and synchrotron x-ray diffraction studies on Weyl semimetals NbAs and TaAs have been carried out along with density functional theoretical (DFT) analysis to explain pressure induced structural and electronic topological phase transitions. The frequencies of first order Raman modes harden with increasing pressure, exhibiting a slope change at $\mathrm{P}_{c}^{N b} \sim 15 \mathrm{GPa}$ for $\mathrm{NbAs}$ and $\mathrm{P}_{c}^{T a} \sim 16 \mathrm{GPa}$ for TaAs. The resistivities of NbAs and TaAs exhibit a minimum at pressures close to these transition pressures and also a change in the bulk modulus is observed. Our first-principles calculations reveal that the transition is associated with an electronic Lifshitz transition at $\mathrm{P}_{c}^{N b}$ for NbAs while it is a structural phase transition from body centered tetragonal to hexagonal phase at $\mathrm{P}_{c}^{T a}$ for TaAs. Further, our DFT calculations show a structural phase transition at $24 \mathrm{GPa}$ from body centered tetragonal phase to hexagonal phase.
\end{abstract}




\section{Introduction}

Weyl semimetals (WSMs) are novel topological materials whose low energy excited states host massless Weyl Fermions. WSMs have two non-degenerate bands crossing each other at isolated points (called Weyl nodes) in Brillouin zone[1]. The Weyl nodes come in pairs of opposite chirality and act as magnetic monopole. Another characteristics of WSMs is the topological surface state characterized by Fermi $\operatorname{arcs}[2,3,4]$ and exotic topological chiral transport properties such as chiral anomaly $[5,6,7,8,9]$ and a large magnetoresistance[10, 11, 12]. WSMs can only be found in materials with broken time-reversal or inversion symmetry. If the time-reversal and inversion symmetries are preserved, Weyl nodes in WSMs become degenerate and form another topological state called Dirac semimetals. Recently, the non-centrosymmetric materials TaAs, TaP, NbAs, and NbP have been predicted to host the WSM state with twelve pairs of Weyl nodes[1, 13]. Soon after the theoretical predictions, the presence of Weyl points[3, 4, 14, 15, 16], Fermi arcs[2, 3, 4, 15] and negative magneto-resistance[10, 11] have been experimentally observed in these compounds.

High pressure is a powerful tool to tune the electronic structure as well as lattice of the material. It is interesting to ask whether pressure can induce some other exotic structures and properties in these topological WSMs. Indeed, the high pressure synchrotron study up to 53 GPa of TaAs [17] shows that TaAs transforms to hexagonal $(\mathrm{P} \overline{6} \mathrm{~m} 2)$ phase at $14 \mathrm{GPa}$ from the ambient $\left(\mathrm{I}_{1} \mathrm{md}\right)$ phase, along with significant changes in the electronic states. Although the high pressure hexagonal phase is also a Weyl semimetal, it has only one set of Weyl points (isoenergetic 12 Weyl points) in contrast to the ambient $\mathrm{I}_{1}$ md phase where two sets of Weyl points ( 4 pairs named as W1 are at $\mathrm{k}_{z}=\mathrm{O}$ plane and other 8 pairs named as W2 are off this plane) separated in energy by $14 \mathrm{meV}$ are present [17]. Interestingly, the Weyl points in $\mathrm{I} 4_{1} \mathrm{md}$ phase move with pressure while in the high pressure hexagonal phase, the Weyl points remain unchanged with pressure [17]. Further high pressure magneto-transport experiments in NbAs upto 2.3 GPa [18] shows that the Fermi surfaces exhibit an anisotropic evolution under pressure. Similarly, high pressure magneto-transport studies up to $2.8 \mathrm{GPa}$ of $\mathrm{NbP}$ [19] show a significant effect of pressure on the amplitudes of Shubnikov-de Haas oscillations, attributed to the subtle changes in the shape of the Fermi surface. The effect of pressure on lattice vibrations and electrical transport of $\mathrm{NbAs}$ and TaAs have not been studied. Here, we report Raman, x-ray diffraction and electrical transport of NbAs and TaAs under pressure together with first-principles density function theoretical calculations to gain microscopic insight of the pressure induced changes. Our main results are: (i) The first order Raman modes of NbAs show slope $(\mathrm{S}=\mathrm{d} \omega / \mathrm{dP})$ change in their phonon frequencies at $\mathrm{P}_{c}^{N b} \sim 15 \mathrm{GPa}$; (ii) The unit cell volume of NbAs obtained from synchrotron x-ray data as function of pressure shows a change in the bulk modulus at $\mathrm{P}_{c}^{N b}$ without any change in the structure; (iii) The resistivity of NbAs decreases with pressure and then starts to increase at pressure close to $\mathrm{P}_{c}^{N b}$; (iv) The phonon frequencies of all the Raman modes of TaAs exhibit a slope change at $\mathrm{P}_{c}^{T a} \sim 16 \mathrm{GPa}$; (v) In contrast to NbAs, x-ray 
data on TaAs show a structural phase transition at $\mathrm{P}_{c}^{T a}$ in agreement with the previous study[17]; (vi) The pressure dependent resistivity of TaAs passes through a minimum at $\mathrm{P}_{c}^{T a}$; (vii) Our DFT calculations reveal that there is pressure induced Lifshitz transition at $\mathrm{P}_{c}^{N b}$ in NbAs and phonon frequencies of Raman modes and resistivity of NbAs bear the signatures of this Lifshitz transition in terms of changes in $\mathrm{S}$ and anomaly in resistivity, respectively. Further, our calculations capture the changes in $\mathrm{S}$ of different Raman modes at $\mathrm{P}_{c}^{T a}$. These results bring out the differences in pressure behavior of two similar Weyl semimetals NbAs and TaAs.

\section{Experimental details}

Raman experiments were performed on single crystals of NbAs and TaAs, grown via chemical vapour transport[20]. Raman measurements were carried out at room temperature in back scattering configuration with micro-optical system equipped with Horiba 800 spectrometer, peltier cooled CCD detector and $532 \mathrm{~nm}$ diode laser. The high pressure synchrotron x-ray diffraction measurements were done at DESY (beam line P02) and Elettra (beam line Xpress) using $0.2888 \AA$ and $0.4957 \AA$ x-ray radiation, respectively. In Raman experiments, thin platelets of dimension $\approx 100 u m$ of NbAs and TaAs were embedded in a 4:1 methanol: ethanol pressure transmitting medium along with ruby chip for pressure calibration into a stainless steel gasket inserted between the diamonds in a diamond anvil cell. For high-pressure electrical resistivity measurements, a diamond anvil cell with a $500 \mu \mathrm{m}$ culet was used. The tungsten gasket was insulated with a cubic BN/epoxy mixture. Single crystal sample of suitable size ( $120 \mu \mathrm{m}$ x 120 $\mu \mathrm{m} \times 10 \mu \mathrm{m})$ was cut and placed into the central hole of the gasket filled with $\mathrm{NaCl}$ as a pressure-transmitting medium along with ruby chip for pressure calibration. The electrical leads were fabricated from $5 \mu \mathrm{m}$ thick Pt foil and attached to the sample in van der Pauw configuration. Resistance was measured at room temperature using a direct current source and nanovoltmeter. Resistivity was calculated taking into account the change of sample thickness upon compression, which was estimated from the experimental equation of state.

\section{Computational details}

Our first-principles quantum mechanical calculations are based on density functional theory (DFT) as implemented in the QUANTUM ESPRESSO (QE) [21] code that replaces the interaction between the core and valence electrons with a smooth pseudopotential (PP). We used fully relativistic ultrasoft pseudopotentials [22] to include spin-orbit coupling (SOC) and treated the exchange-correlation energy of the electrons with a functional in local density approximation (LDA) as parametrized by Perdew, and Zunger [23]. The PPs were constructed with valence configurations $4 \mathrm{~s}^{2} 5 \mathrm{~s}^{2} 5 \mathrm{p}^{6} 4 \mathrm{~d}^{3}$, $5 \mathrm{~s}^{2} 6 \mathrm{~s}^{2} 6 \mathrm{p}^{6} 5 \mathrm{~d}^{3}$ and $4 \mathrm{~s}^{2} 4 \mathrm{p}^{3}$ for $\mathrm{Nb}$, Ta and As atoms, respectively. While performing self-consistent field (SCF) calculations, we truncated the plane wave basis with cut-off 
energies of 60 Ry and 600 Ry in representation of Kohn-Sham wave functions and charge density, respectively. The discontinuity in occupation numbers of the electronic states at the Fermi level was smeared with an energy width of $k_{B} T=0.003$ Ry in the Fermi-Dirac distribution function. The Brillouin zone integrations were sampled on uniform mesh of $12 \times 12 \times 12$ k-vectors. Fermi surfaces are interpolated on a dense $33 \times 33 \times 33$ grid of k-point in the Brillouin zone. We calculated the phonon frequencies of NbAs and TaAs using density functional perturbation theory (DFPT) as implemented in the PH module [24] in the QE codes. To obtain phonon dispersion, we first determined the force constant matrices on a $2 \times 2 \times 2$ mesh of q-points. The dynamical matrices at arbitrary q-points are then obtained using Fourier interpolation of these and diagonalized to determine the phonon frequencies. Comparison of phonon dispersion calculated with and without including spin-orbit coupling (SOC) reveals (see Fig.9 (c,d)) that the changes in phonon frequencies are quite small $\left(<1 \mathrm{~cm}^{-1}\right)$. Therefore, we determined the phonon frequencies of $\mathrm{NbAs}$ and TaAs using scalar relativistic ultrasoft pseudopotentials (that do not include the SOC) as a function of hydrostatic pressure. Electronic and vibrational properties at each target pressure were determined at fully optimized crystal structures of NbAs and TaAs with the inclusion of SOC in self-consistent field calculations until the forces on each atom becomes less than $1 \mathrm{mRy} /$ bohr. To determine the bulk modulus (B) for different structures of NbAs and TaAs, we fitted V vs P plot with Birch Murnaghan equation of state.

$$
P(V)=\frac{3 B}{2}\left[\left(\frac{V_{0}}{V}\right)^{\frac{7}{3}}-\left(\frac{V_{0}}{V}\right)^{\frac{5}{3}}\right]\left[1+\frac{3}{4}\left(B^{\prime}-4\right)\left(\left(\frac{V_{0}}{V}\right)^{\frac{2}{3}}-1\right)\right]
$$

where $\mathrm{V}$ is the volume at pressure $\mathrm{P}, \mathrm{V}_{0}$ is the reference volume, $\mathrm{B}$ is the bulk modulus, and $B^{\prime}$ is the pressure derivative of the bulk modulus. All the calculations here are based on the use of the primitive unit cell of these compounds as a periodic unit.

\section{Results and discussion}

\subsection{Experimental Results}

NbAs and TaAs have non-centrosymmetric body centered tetragonal (BCT) structure corresponding to space group I4 $_{1}$ md (109) [25]. The crystal structure of XAs (X=Nb, Ta) has symmetry determined sites (4a Wyckoff site) for X ( $\mathrm{Nb}$ or $\mathrm{Ta}$ ) and As atoms, with slightly distorted (two short and four long X-As bonds) trigonal prismatic coordination environments of each other (i.e. $\mathrm{XAs}_{6}$ or $\mathrm{X}_{6} \mathrm{As}$ ). Sheets of these trigonal prisms extended in the ab-plane and stacked along c-axis form a networked structure. $\mathrm{Nb}(\mathrm{Ta})$ and $\mathrm{As}$ atoms have strong covalent bonding, making it stable and robust under pressure. The conventional (see Fig.1 (a)) and primitive (see Fig.1 (b)) unit cells of XAs (X=Nb, Ta) have eight and four atoms, respectively. According to factor group analysis, this system has $\left[\mathrm{A}_{1}+\mathrm{E}\right]$ acoustic phonons and $\left[\mathrm{A}_{1}+2 \mathrm{~B}_{1}+3 \mathrm{E}\right]$ optical phonons. All the optical phonons are Raman active[26]. The Raman spectra of NbAs at a few representative pressures are shown in Fig.2 (a). Five Raman modes were observed and assigned to 
$\mathrm{E}^{1}, \mathrm{~B}_{1}^{1}, \mathrm{~B}_{1}^{2}, \mathrm{E}^{2}$ and $\mathrm{A}_{1}$ irreducible representations by comparing the data with firstprinciples calculations. In order to find out the phonon frequencies and full width at half maximum (FWHM) of the Raman modes, Lorentzian line shapes were fitted to the Raman spectra after applying polynomial background correction (red lines in Fig.2 (a)). Fig.3 (a) shows pressure dependence of phonon frequencies of $\mathrm{E}^{1}, \mathrm{~B}_{1}^{1}, \mathrm{~B}_{1}^{2}, \mathrm{E}^{2}$ and $\mathrm{A}_{1}$ modes. It is clear from Fig.3(a) that the observed phonon frequencies of all the Raman modes except $\mathrm{E}^{1}$ exhibit a change in the slope $\mathrm{S}(=d \omega / d P)$ at $\mathrm{P}_{c}^{N b} \sim 15 \mathrm{GPa}$, signifying a phase transition. We note that the slope change for the $\mathrm{B}_{1}^{1}$ modes is higher than other modes. This transition is further investigated by pressure dependent resistivity measurements (Fig.4 (a)). The resistivity decreases with increasing pressure till $\sim 12$ GPa, exhibiting normal pressure dependence. However, the pressure dependence of the resistivity becomes anomalous after $\sim 12 \mathrm{GPa}$ : it starts to increase with increasing pressure. This signifies the change in the electronic structure after $12 \mathrm{GPa}$. In another study by M. Einaga et al. [27] on the same family of Weyl semimetals NbP and TaP, similar trend was observed and the anomalous behavior of resistivity was associated with the decrease of density of states corresponding to Weyl points, located around the Fermi energy. The reason for the slight difference between the transition pressure in resistivity data and $\mathrm{P}_{c}^{N b}$ might be due to the enhanced sensitivity of the former to changes in the electronic structure (to be discussed latter) as compared to the phonon frequencies. Thus our Raman and resistivity results reveal a phase transition at $\mathrm{P}_{c}^{N b}$. To find out structural changes with pressure, we performed high pressure synchrotron x-ray diffraction experiments using $0.288 \AA$ x-ray wavelength. Fig.5 (a) shows the pressure dependence of synchrotron x-ray diffraction patterns at a few representative pressures. This clearly reveals that the number of diffraction peaks remain same till $25 \mathrm{GPa}$, ruling out the possibility of structural phase transition at $\mathrm{P}_{c}^{N b}$ as to be confirmed latter using DFT. In order to get the lattice parameters shown in Fig.5 (b), we did Rietveld refinement using Jana 2006[28]. We used I4 ${ }_{1}$ md space group for the refinement of the unit cell at different pressures. Fig.5 (c) shows pressure dependence of unit cell volume. The solid red lines are fit to Eq. (1). It is clear from Fig.5 (c) that the bulk modulus (B) changes at $\mathrm{P}_{c}^{N b}$ (indicated by vertical dashed line), suggesting a phase transition. Thus high pressure x-ray data rules out a structural phase transition at $\mathrm{P}_{c}^{N b}$ but a significant change in B does suggest an isostructural electronic phase transition [29, 30, 31].

We will now present our experimental results on TaAs. We show Raman spectra of TaAs at a few representative pressures in Fig.2 (b). As mentioned in previous paragraph, the solid red lines are the Lorentzian fit to the experimental data (black lines). Five observed Raman modes were assigned to $\mathrm{B}_{1}^{1}, \mathrm{E}^{2}, \mathrm{~A}_{1}, \mathrm{E}^{3}$ and $\mathrm{B}_{1}^{2}$ irreducible representations based on first-principles calculations. Fig.6 (a) shows the pressure dependence of phonon frequencies of $\mathrm{B}_{1}^{1}, \mathrm{E}^{2}, \mathrm{~A}_{1}, \mathrm{E}^{3}$ and $\mathrm{B}_{1}^{2}$ modes. It is clear from Fig.6 (a) that all the Raman modes harden with increasing pressure and exhibit a change in $\mathrm{S}$ at $\mathrm{P}_{c}^{T a} \sim 16 \mathrm{GPa}$. The strongest Raman mode $\mathrm{A}_{1}$ shows very large deviation at $\mathrm{P}_{c}^{T a}$ and doest not follow linear behavior beyond $\mathrm{P}_{c}^{T a}$. The Raman modes $\mathrm{B}_{1}^{1}, \mathrm{E}^{3}$ and $\mathrm{E}^{2}$ became too weak to follow after $22 \mathrm{GPa}$ and hence frequencies of these modes are given 


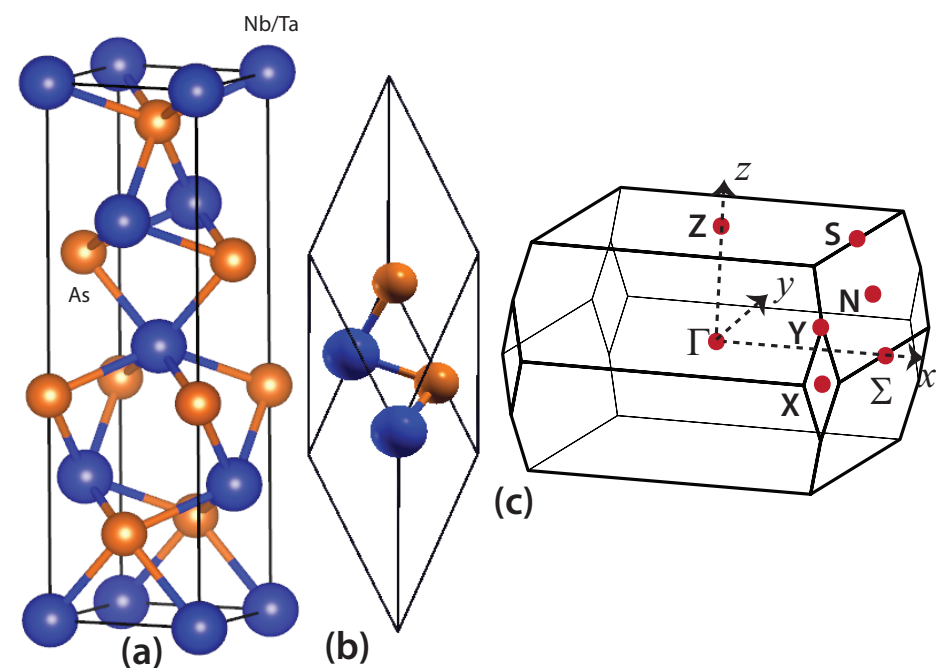

Figure 1. (Color online)(a) Conventional, (b) primitive unit cells and (c) Brillouin zone of the primitive unit cell with high symmetry points marked with red dots. Blue and brown colors indicate $\mathrm{Ta}(\mathrm{Nb})$ and As atoms respectively.

till 22 GPa only. To further investigate this transition, we did high pressure resistivity measurements as shown in Fig.4 (b). It is clear from Fig.4 (b) that resistivity exhibits a change in its trend at $\sim 14 \mathrm{GPa}$ : it decreases first and then starts to increase at $\sim 14$ GPa. To examine the structural phase transition, we did high pressure synchrotron x-ray diffraction experiments with $0.4957 \AA$ x-ray wavelength. Fig.7 (a) shows the pressure dependence of the x-ray diffraction patterns of TaAs at a few representative pressures. The intensity of the peak at $2 \theta \sim 15^{\circ}$ decreases with increasing pressure and becomes very weak at $18.5 \mathrm{GPa}$ onwards. However, this peak remains till $26 \mathrm{GPa}$, consistent with previous report[17]. Theoretical calculations (to be discussed latter) predict a structural phase transition from BCT to hexagonal phase at higher pressures. We find that the diffraction peaks of hexagonal phase of TaAs are very close to the BCT phase diffraction peaks and are significantly broadened at higher pressures. So the diffraction peaks of high pressure hexagonal phase are merging with diffraction peaks of BCT phase due to their broadening. Thus lattice parameters were obtained by Rietveld refinement using Jana 2006 [28] with space group I4 ${ }_{1}$ md (for BCT phase) and with a mixture of $\mathrm{I}_{1} \mathrm{md}$ and $\mathrm{P} \overline{\mathrm{b}} \mathrm{m} 2$ (for hexagonal phase) beyond $14 \mathrm{GPa}$ as shown in Fig.7 (b). Our x-ray results are consistent with the earlier report [17]. The pressure dependence of the volume of both the phases are shown in Fig.7 (c).

\subsection{Theoretical calculations}

Our estimates of the optimized lattice constants are as follows: for NbAs, $a=b=3.42 \AA$, $\mathrm{c}=11.53 \AA$ and for TaAs, $\mathrm{a}=\mathrm{b}=3.41 \AA, \mathrm{c}=11.51 \AA$, which agree quite well with their experimental values [32]. Calculated lattice constants of NbAs and TaAs (see Fig.8 (a-b)) vary smoothly as a function of hydrostatic pressure within BCT structure. TaAs shows a structural phase transition (to be discussed latter) at $11.3 \mathrm{GPa}$ and hence 

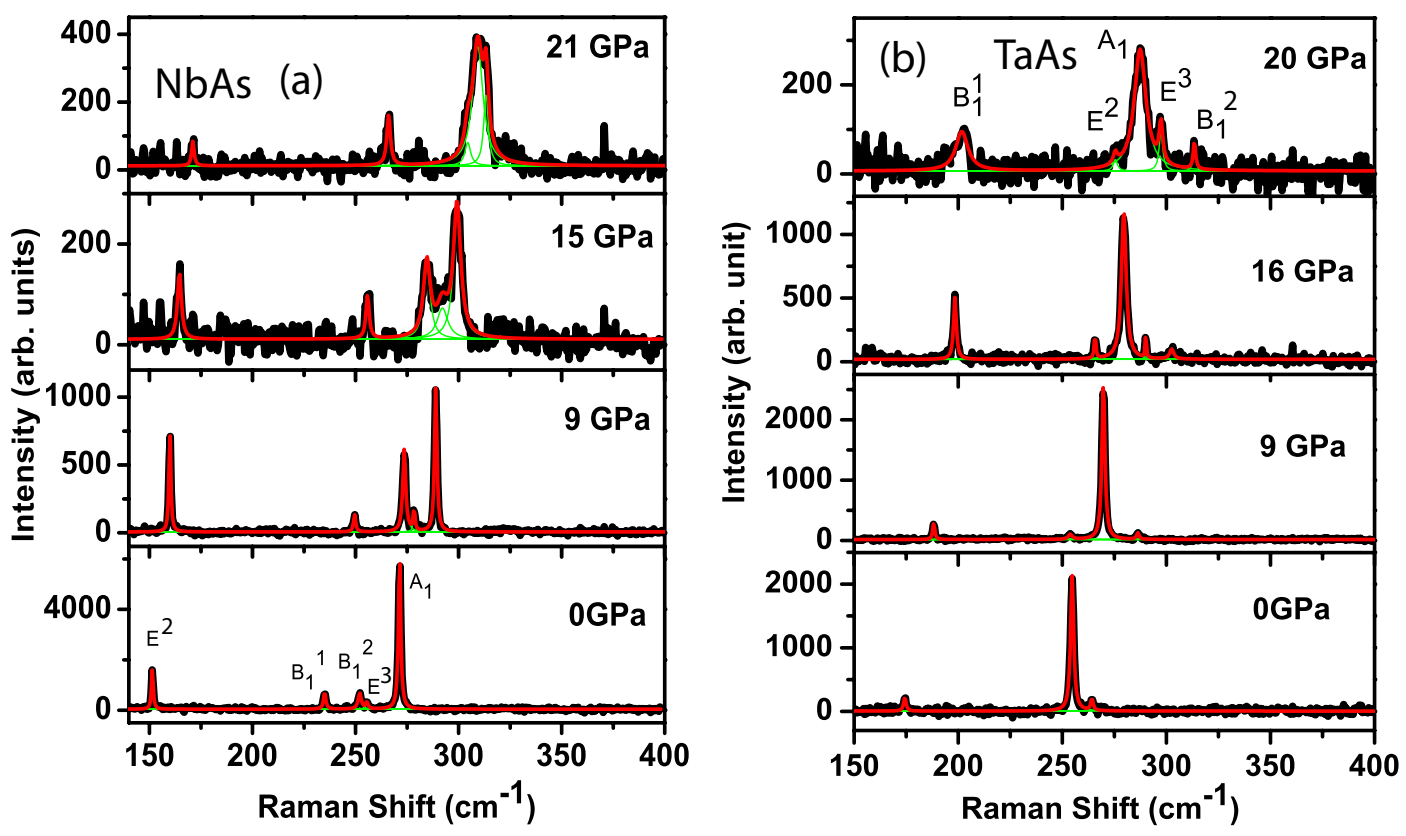

Figure 2. (Color online) Raman spectra of NbAs and TaAs at a few representative pressures. The solid lines (red and green) are the Lorentzian fit to the measured spectrum (black).

pressure dependence of the lattice parameters of the high pressure hexagonal phase are given after $12 \mathrm{GPa}$. Calculated bulk moduli of the BCT structure of NbAs (TaAs) are $188 \pm 2 \mathrm{GPa}(193 \pm 1 \mathrm{GPa})$, which agree with experimental values of $180 \pm 6 \mathrm{GPa}$ (191 $\pm 6 \mathrm{GPa})$. The change in the bulk modulus across the phase transition as observed in the experiment in NbAs is not seen in the theoretical calculation, which might be due to typical DFT errors in c/a ratios of the two phases across the transition. We calculated electronic structure of NbAs (Fig.9 (a)) and TaAs (Fig.9(b)) at their optimized lattice constants (i.e. at $0 \mathrm{GPa}$ ) first without the inclusion of spin-orbit coupling. In the absence of spin-orbit coupling, the highest occupied valence (VBM) and lowest unoccupied conduction (CBM) bands cross at the Fermi level and give rise to nodal rings [1, 33], parts of which can be seen as crossings (see black lines in Fig.9 (a) and (b)) between those bands around the Fermi level along high-symmetry directions $(\Gamma-\Sigma-S-Z-N)$ in the Brillouin zone (see Fig.1 (c) for notation). When the spin-orbit coupling is introduced, the nodal ring is gapped out (band gap opens up at the band crossings, see red lines in Fig.9 (a) and green lines in Fig.9 (b) for NbAs and TaAs, respectively) and Weyl nodes are created [1,33]. Phonon dispersions of these compounds calculated with and without SOC reveal negligible changes in the phonon frequencies (see Fig.9 (c) and Fig.9 (d)). Our calculated frequencies of the Raman active modes are in good agreement with the experiments (see Table I for comparison).

Fig.3 (b) shows the pressure dependence of calculated phonon frequencies of Raman active modes of NbAs. It is clear from Fig.3 (b) that all the Raman modes show change in $\mathrm{S}$ at $\mathrm{P}_{c}^{N b \text { theory }} \sim 16 \mathrm{GPa}$, consistent with the experimental results. The difference 

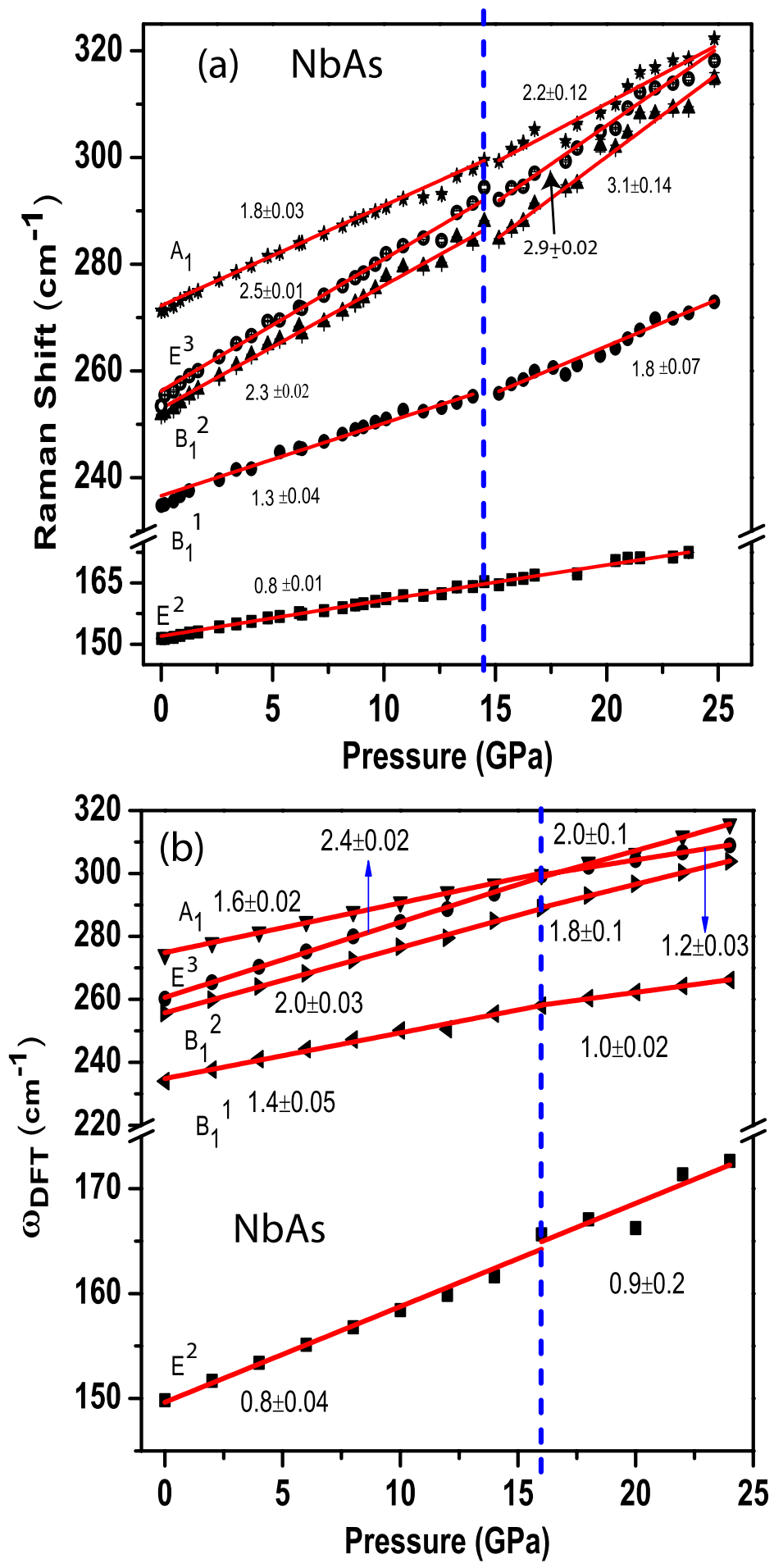

Figure 3. (Color online) Pressure dependence of phonon frequencies of NbAs: (a) Experimentally observed, (b) Theoretically calculated. The solid lines are the linear fit to the experimental data. The slope $\mathrm{S}$ in the unit of $\mathrm{cm}^{-1} / \mathrm{GPa}$ is given near the lines. The vertical dashed lines indicates the phase transition pressures. 
Pressure-induced Lifshitz and structural transitions in NbAs and TaAs: Experiments and Theory9
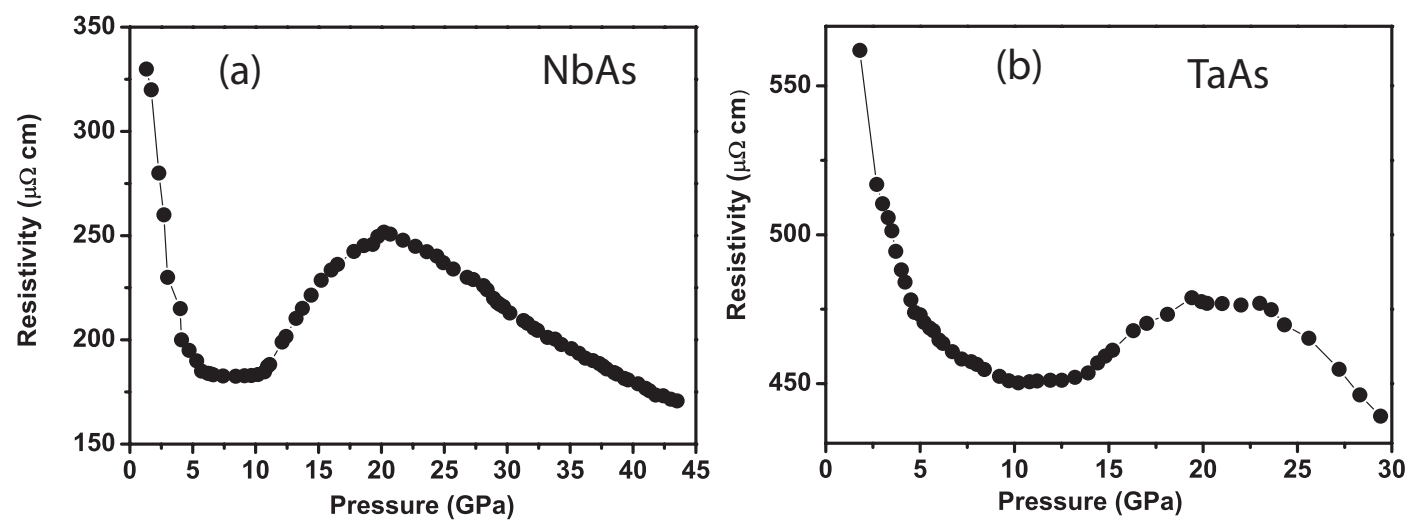

Figure 4. (Color online) Pressure dependence of measured resistivity of (a) NbAs and (b) TaAs.
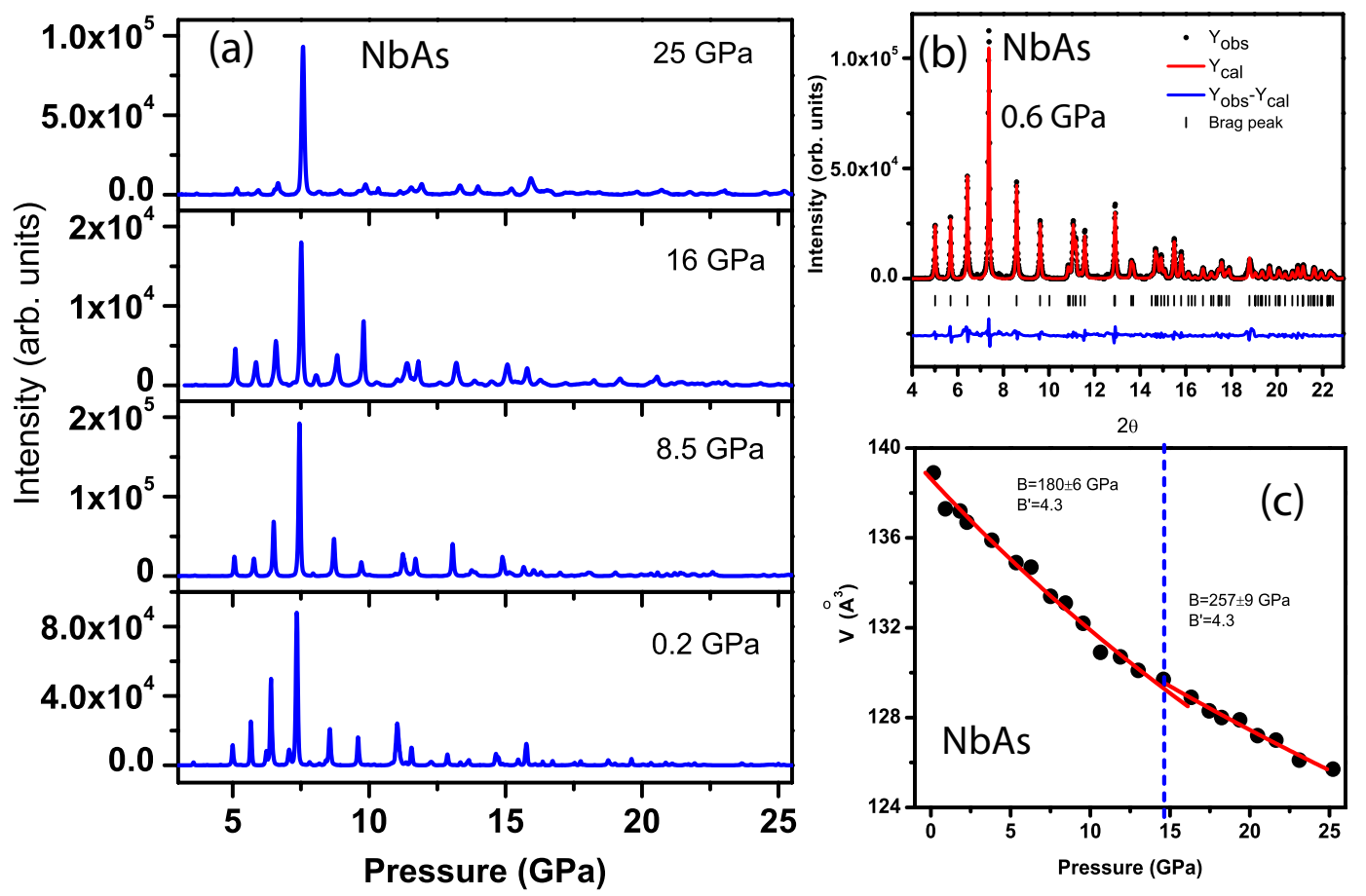

Figure 5. (Color online) (a) X-ray diffraction pattern of NbAs as a function of pressure. (b) The Rietveld refinement of X-ray diffraction pattern at 0.6 GPa pressure. (c) Pressure dependence of volume of NbAs. The vertical dashed line indicates the phase transition pressure. 

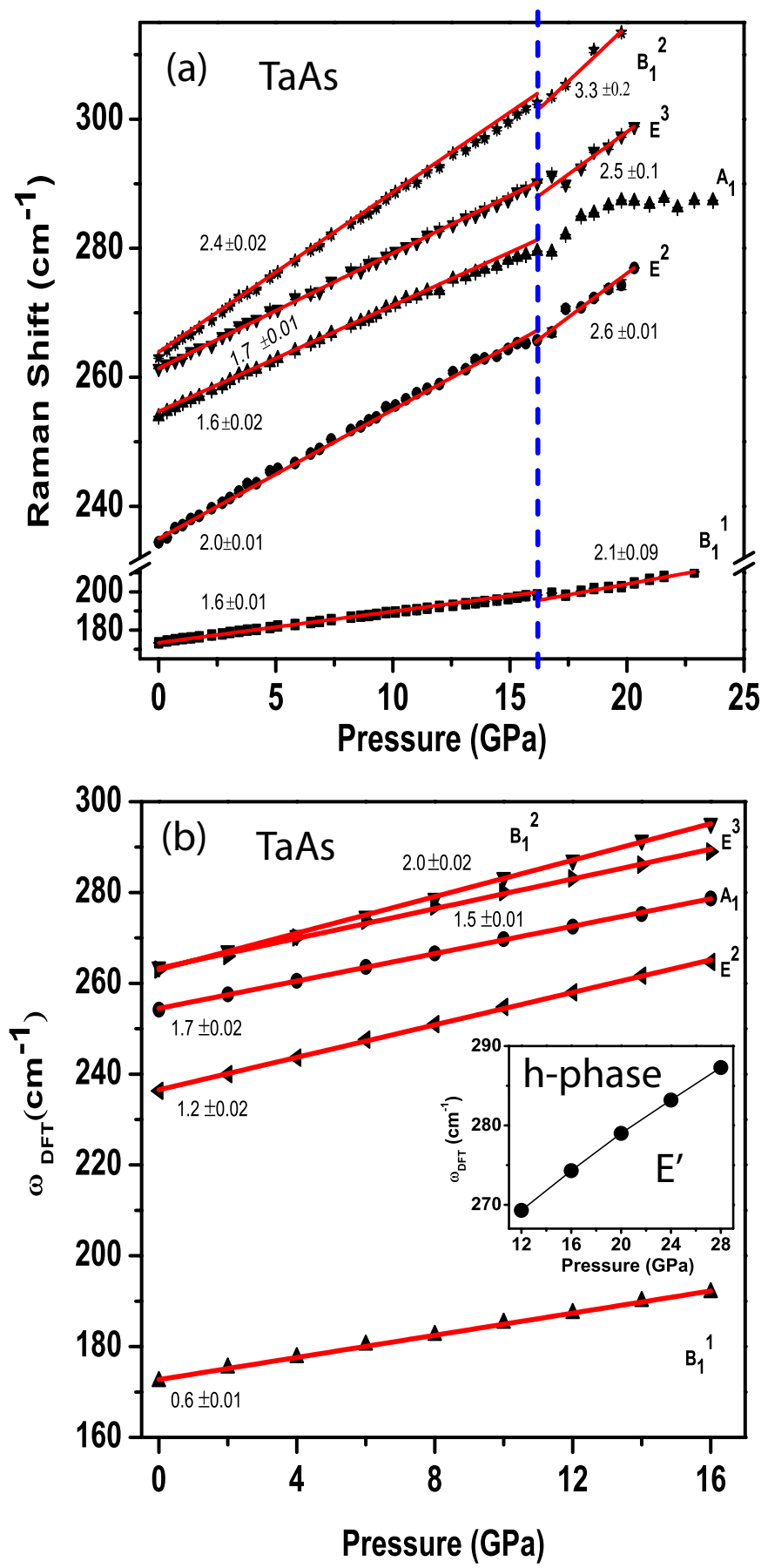

Figure 6. (Color online) Pressure dependence of phonon frequencies of TaAs (a) Experimentally observed, (b) Theoretically calculated. The solid lines are the linear fit to the experimental data. The slope $\mathrm{S}$ in the unit of $\mathrm{cm}^{-1} / \mathrm{GPa}$ is given near the lines. The vertical dashed line indicates the phase transition pressure. Calculated Phonon frequencies of the hexagonal phase of TaAs at higher pressures are shown in the inset of (b). 

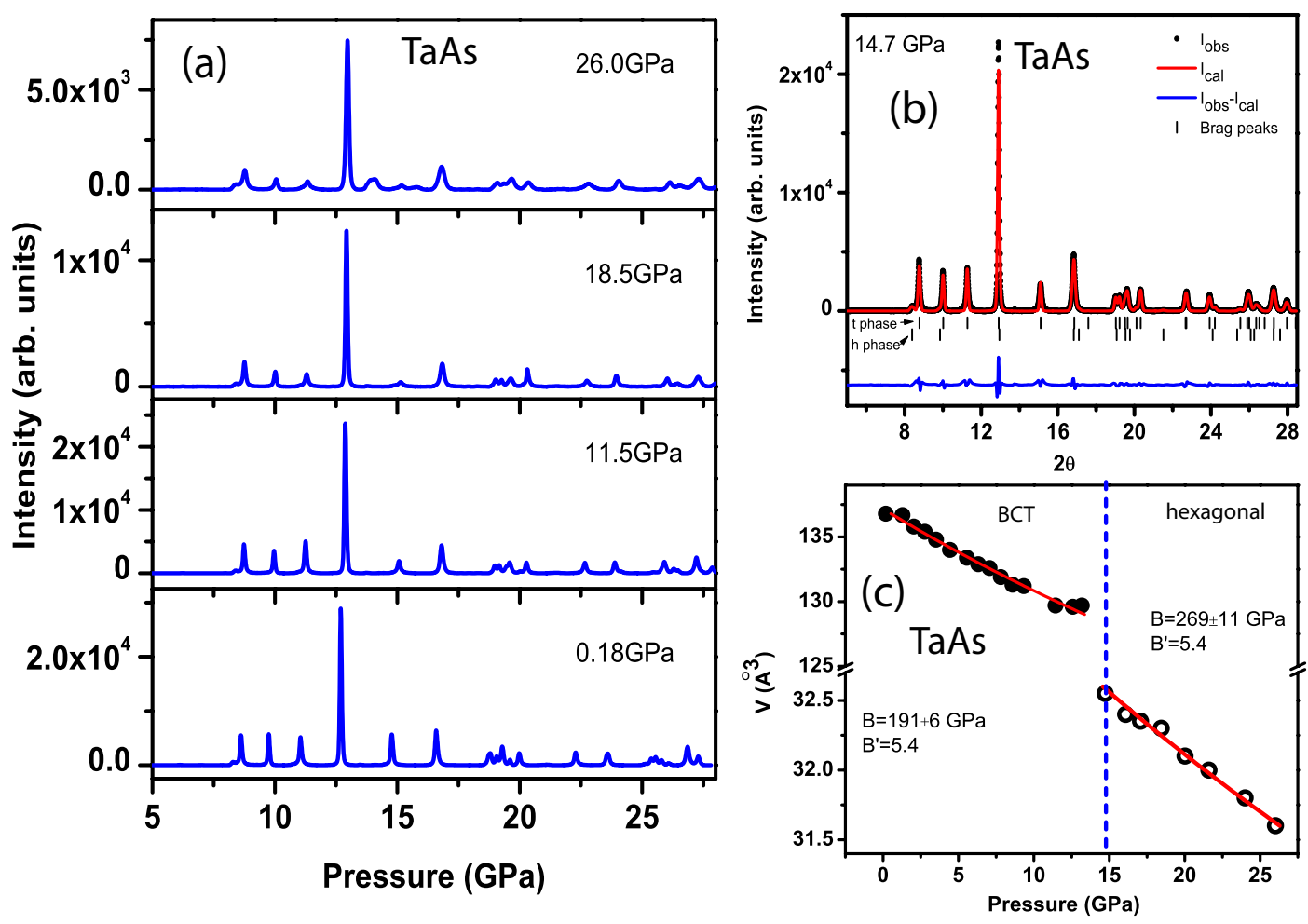

Figure 7. (Color online) (a) X-ray diffraction pattern of TaAs as a function of pressure. (b) The two phase Rietveld refinement of x-ray diffraction pattern at 14.7 GPa. (c) Change in volume of TaAs as a function of pressure. The vertical dashed line indicates the phase transition pressure.

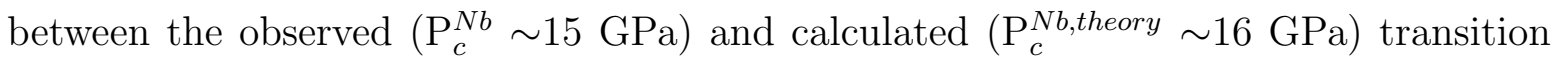
pressures is within the errors of DFT. We note that the calculated pressure dependence of $\mathrm{A}_{1}$ and $\mathrm{E}^{2}$ mode frequencies show similar trends of the pressure coefficients as in experiment across the transition. Discrepancy between theory and experiments on the changes in the pressure coefficients of $\mathrm{E}^{3}, \mathrm{~B}_{1}{ }^{1}, \mathrm{~B}_{1}{ }^{2}$ modes across the transition can be traced to that in the changes in bulk modulus. Such errors in the bulk modulus may arise from the typical DFT errors in c/a ratios of the two phases across the transition. To investigate the pressure dependent structural phase transitions to hexagonal (P-6m2) or cubic (Pm-3m) phase, we estimated the changes in enthalpy $(\Delta \mathrm{H})$ of these structures and the BCT ( $\mathrm{I}_{1} \mathrm{md}$ ) structure. Fig.12 (a) shows that $\Delta \mathrm{H}$ of the hexagonal structure and BCT decreases with pressure and attain a negative value at $24 \mathrm{GPa}$. Thus, our calculations predict structural phase transition at $24 \mathrm{GPa}$ from the BCT to hexagonal structure. It is rather tempting to associates the non-monotonic behavior of electrical resistivity in NbAs (Fig.4 (a)) near $\sim 22 \mathrm{GPa}$ with this structural transition. Similarly, $\Delta \mathrm{H}$ of cubic phase and BCT structure decreases monotonically with pressure, but it does not become negative till $24 \mathrm{GPa}$ (Fig.12 inset), ruling out a transition to the cubic (Pm-3m) phase as well till $24 \mathrm{GPa}$. Our conclusion of absence of structural phase transition at $16 \mathrm{GPa}$ is consistent with our x-ray results.

To explore further, we analyze the evolution of electronic structure with pressure 
Table 1. Comparison of the observed and calculated frequencies of Raman active modes of NbAs and TaAs at $0 \mathrm{GPa}$. Excellent agreement between theory and experiment is evident.

\begin{tabular}{ccc|ccc}
\hline & \multicolumn{2}{c|}{ NbAs $(0 \mathrm{GPa})$} & \multicolumn{3}{c}{ TaAs $(0 \mathrm{GPa})$} \\
\hline Modes & Experiment & Theory & Modes & Experiment & Theory \\
\hline$E^{2}$ & 151 & 150 & $B_{1}^{1}$ & 173 & 172 \\
$B_{1}^{1}$ & 234 & 234 & $E^{2}$ & 233 & 235 \\
$B_{1}^{2}$ & 252 & 256 & $A_{1}$ & 254 & 255 \\
$E^{3}$ & 253 & 260 & $E^{3}$ & 261 & 260 \\
$A_{1}$ & 271 & 274 & $B_{1}^{2}$ & 264 & 262 \\
\hline
\end{tabular}

to understand the origin of observed changes in Raman frequencies seen at $\mathrm{P}_{c}^{\text {Nb,theory }}$ (Fig.3). At ambient pressure, NbAs is semimetallic in nature, exhibiting tiny electron (hole) pockets along $\Gamma-\Sigma(\Sigma-\mathrm{N})$ lines (Fig.10 (a)). With applied hydrostatic pressure, electronic energy levels do not split. However, energy bands near the Fermi level change considerably with pressure involving transfer of electrons from one pocket to another pocket to maintain the total number of carriers [34]. There are small changes in size of the electron and hole pockets observed at higher pressure (Fig.10 (b, c, d)). We observe considerable change in the shape of the bands (valence band maximum and conduction band minimum) along $\Sigma-S$ path (Fig.10) and in the vicinity of N-point. To understand this further, we examine the evolution of Fermi surface with pressure. Fermi surface contains both electron and hole pockets at 0 GPa (Fig.11 (a)), but electron pockets are very tiny (along $\Gamma-\Sigma$ path, see Fig.10 (a)). Electron and hole pockets are almost semicircular and distributed along nodal rings on $\mathrm{k}_{x}=0$ and $\mathrm{k}_{y}=0$ planes in the BZ. At $12 \mathrm{GPa}$, electron pockets disappear in vicinity of $\Sigma$-point and a new electron pocket appears (in between existing hole pocket in the vicinity of N-point) together with the increase in the size of existing hole pocket at N-point (Fig.11 (c)). The proposed Lifshitz transition at $\mathrm{P} \sim 16 \mathrm{GPa}$ in NbAs is based on the changes in size and shape of electron and hole pockets with pressure. In Fig.11 (c) and Fig.11 (d), we clearly see the difference in the size of the hole pockets (reduces in Fig. Fig.11 (d)) and electron pockets (increases in Fig.11 (d)) at the center of the semicircular arcs of the Fermi surface at $\mathrm{k}_{z} \neq 0$ plane. Also, changes in the shape of hole pockets at the edges of the semicircular arcs are notable. These modifications in the Fermi surface with pressure reveal a clear change in its topology marking a Lifshitz transition at P $16 \mathrm{GPa}$. This is correlated with changes in calculated pressure coefficients of Raman active modes and can be responsible for the changes in resistivity at $\mathrm{P}_{c}^{N b}$.

We now discuss our results on TaAs. Fig.6 (b) shows pressure dependent evolution of the phonon modes of TaAs. Our calculations reveal that there is no change in $\mathrm{S}$ up to $16 \mathrm{GPa}$, which is consistent with our experimental observations. To detect the presence of any structural phase transition, we considered two high symmetry crystal 
Pressure-induced Lifshitz and structural transitions in NbAs and TaAs: Experiments and Theory 13
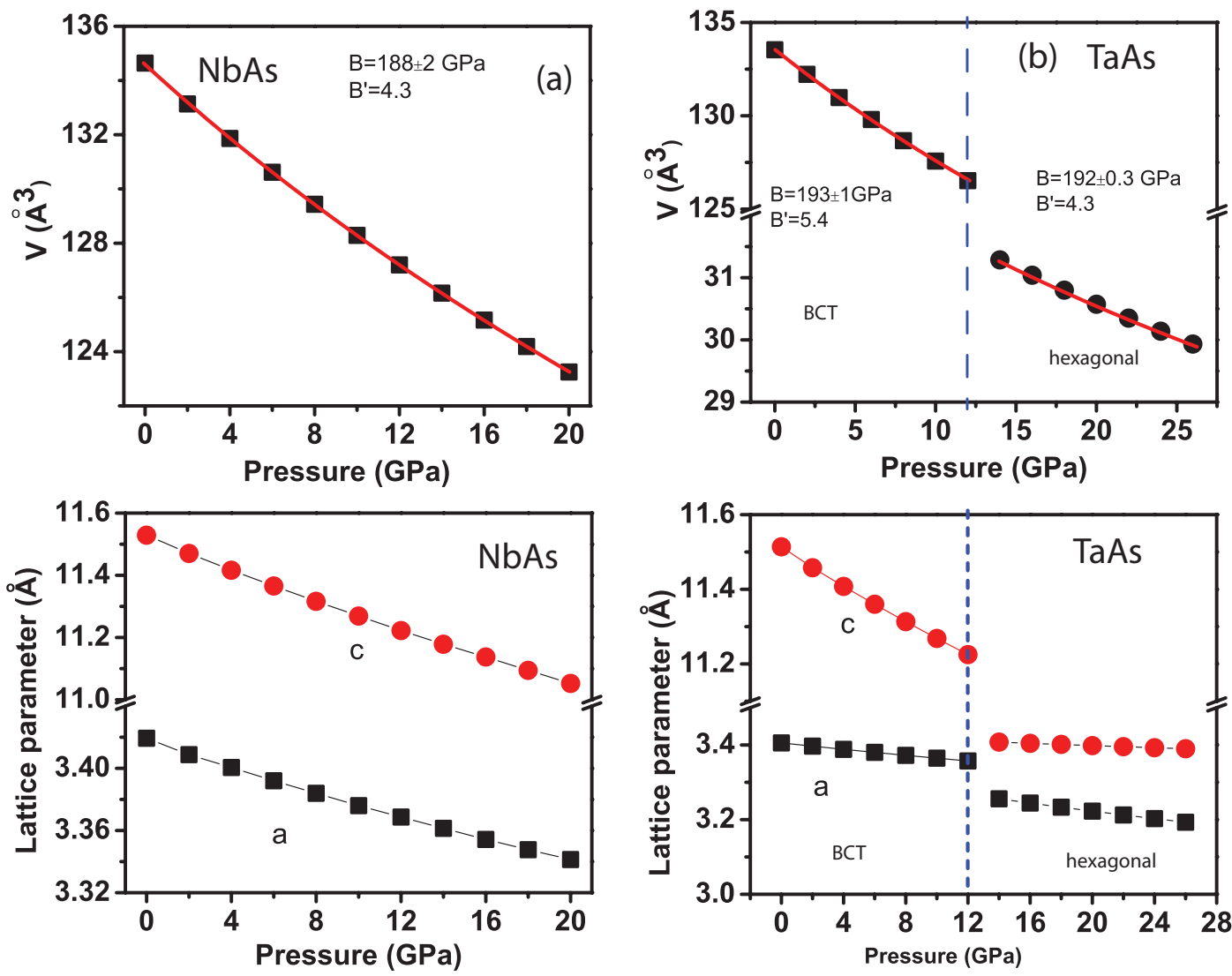

Figure 8. (Color online)-Lattice constants of NbAs (a) and TaAs (b) along with their volume as a function of hydrostatic pressure.

Table 2. Optimized lattice parameters of the hexagonal crystal structure of TaAs at $\mathrm{P}=0 \mathrm{GPa}$ and at $\mathrm{P}=14 \mathrm{GPa}$. The atomic positions are in fractional coordinates.

Hexagonal unit cell (No. of atoms $=2$ )

\begin{tabular}{cccc}
\hline Atom & $\mathrm{x}$ & $\mathrm{y}$ & $\mathrm{z}$ \\
\hline $\mathrm{Ta}$ & 0 & 0 & 0 \\
As & $\frac{2}{3}$ & $\frac{1}{3}$ & $\frac{1}{2}$ \\
Lattice constants (at 0 GPa) & $\mathrm{a}=3.35 \AA, \quad \mathrm{c}=3.44 \AA$ & \\
Lattice constants (at 14 GPa) & $\mathrm{a}=3.26 \AA, \quad \mathrm{c}=3.41 \AA$ & \\
\hline
\end{tabular}

structures : (a) cubic (CsCl structure with space group Pm-3m, \# 221 and (b) hexagonal (WC structure with space group P-6m2, \# 162, and calculated the enthalpy differences between these phases and the BCT structure. We find that that while the enthalpy difference $(\Delta H)$ of the cubic structure decreases with pressure, it does not become negative until $24 \mathrm{GPa}$ (Fig.12 (b) inset). On the other hand, $\Delta H$ of the hexagonal phase and the BCT structure decreases monotonously with pressure and becomes negative above $11 \mathrm{GPa}$ (Fig.12 (b)). Clearly, a structural phase transition from the BCT phase to the hexagonal phase is predicted to occur at $10.8 \mathrm{GPa}$ at $0 \mathrm{~K}$, see structural information 

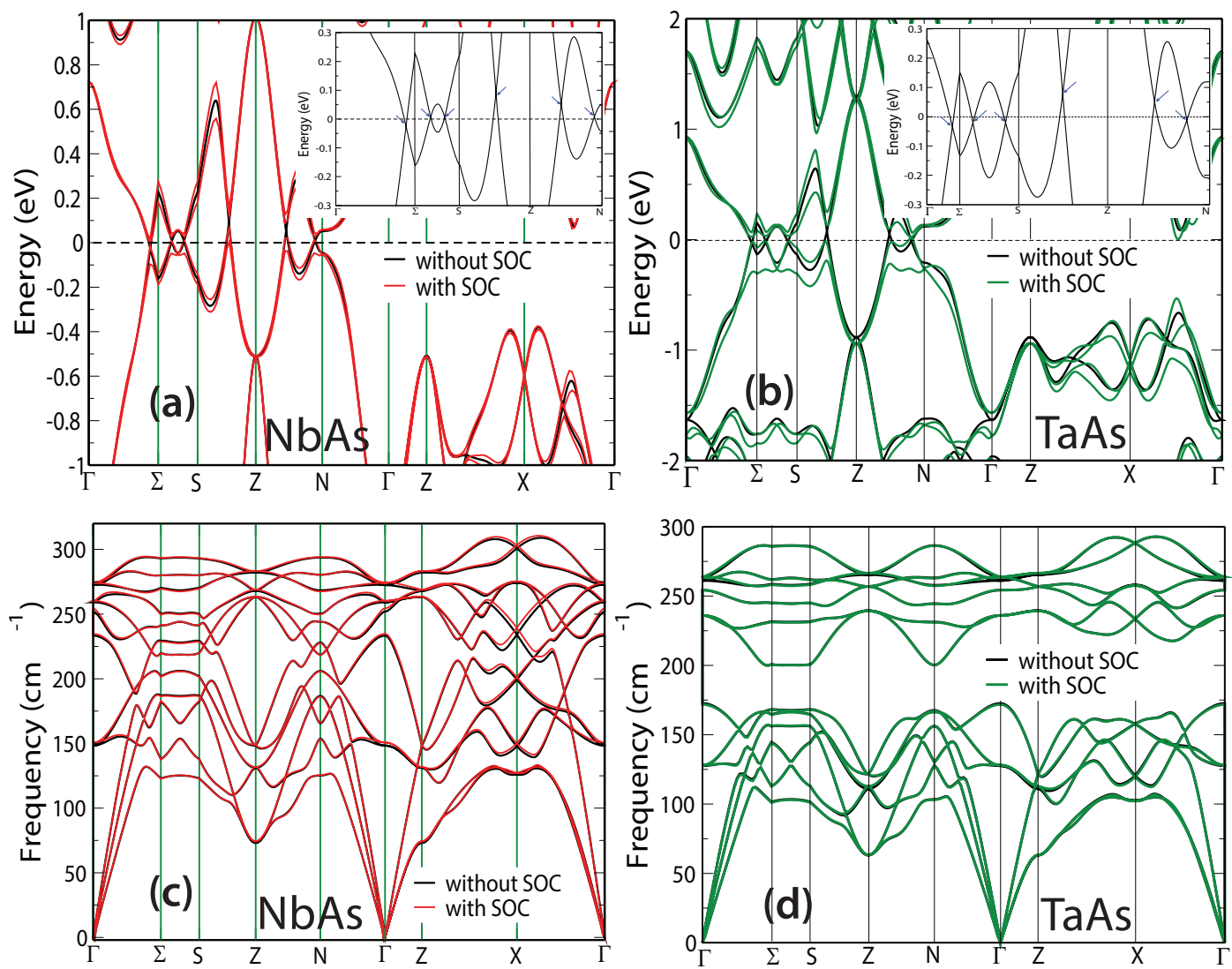

Figure 9. (Color online) (a) Electronic structure of (a) NbAs and (b) TaAs calculated with and without SOC. Zoomed-in electronic structure (calculated without SOC) along $\Gamma-\Sigma-\mathrm{S}-\mathrm{Z}-\mathrm{N}$ is given in the inset to show the band crossing points (marked with blue arrow) that are part of the nodal rings in the Brillouin zone. Phonon dispersion of (c) NbAs and (d) TaAs calculated with and without SOC.

on the hexagonal phases of TaAs in Table II). To predict the transition pressure at $300 \mathrm{~K}$, we calculated the vibration contribution to the free energy using the formula: $\mathrm{F}_{\mathrm{vib}}=\frac{k_{B} T}{N_{q}} \sum_{i q} \log \left[2 \sinh \left(\frac{\hbar \omega_{i q}}{2 k_{B} T}\right)\right]$. Here $N_{q}$ is the total number of wave vectors $q$ in the Brillouin zone, $\omega_{i q}$ is the frequency of $i$-th phonon with wave vector $q$ obtained using DFT linear response calculations. The difference Gibbs' free energy $(\Delta \mathrm{G}=\Delta \mathrm{H}$ $\left.(\mathrm{T}=0)+\mathrm{F}_{v i b, h e x}-\mathrm{F}_{v i b, B C T}\right)$ between the hexagonal and BCT structure is given at two representative pressures in Fig.12 (c) as a function of temperature. Interpolation of $\Delta \mathrm{G}$ predicts that the transition pressure $\left(\mathrm{P}_{c}^{\text {Ta,theory }}\right)$ at $11.3 \mathrm{GPa}$ at $300 \mathrm{~K}(\mathrm{Fig} .12(\mathrm{~d}))$. We calculated the phonon dispersion for the hexagonal structure of TaAs at $0 \mathrm{GPa}$ (Fig.12 (e)) and 14 GPa (Fig.12 (f)), which do not exhibit any negative frequency. This signifies its structural stability at $\mathrm{P}>11.3 \mathrm{GPa}$. Thus the slope change in the Raman active modes and anomaly in resistivity data at $\mathrm{P}_{c}^{T a}$ is associated with structural phase transition at $\mathrm{P}_{c}^{\text {Ta,theory }} \sim 11.3 \mathrm{GPa}$. The difference between the transition pressures $\left(\mathrm{P}_{c}^{\text {Ta,theory }}=11.3 \mathrm{GPa}\right.$ in theory vs. $\mathrm{P}_{c}^{T a}=16 \mathrm{GPa}$ in experiments $)$ may be due to (a) errors in calculate lattice constants and (b) deviation from the hydrostatic pressures may be involved in experiment at higher pressures. We have estimated the phonon frequencies 


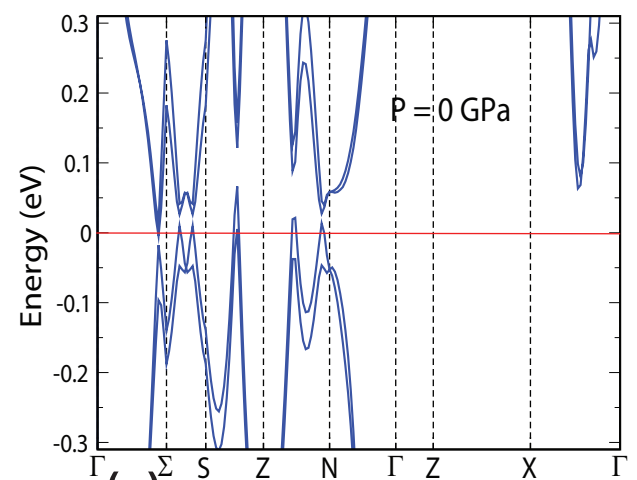

(a)

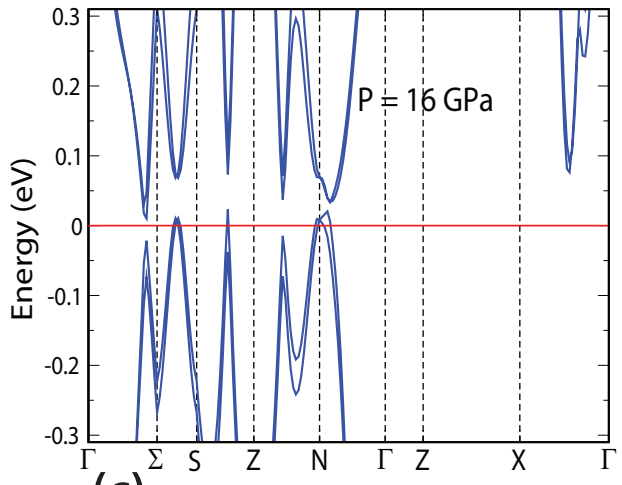

(c)

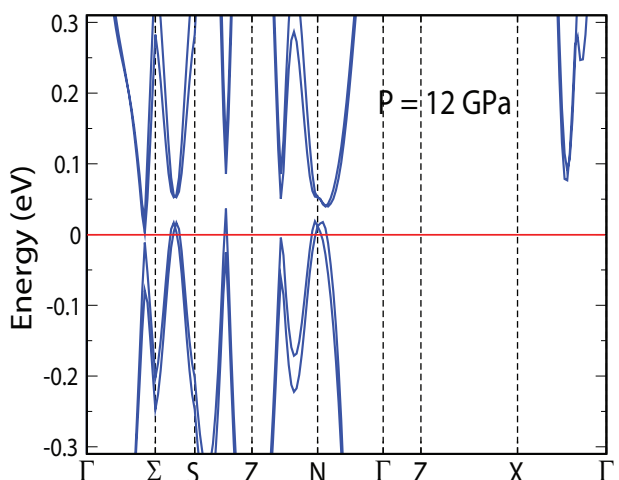

(b)

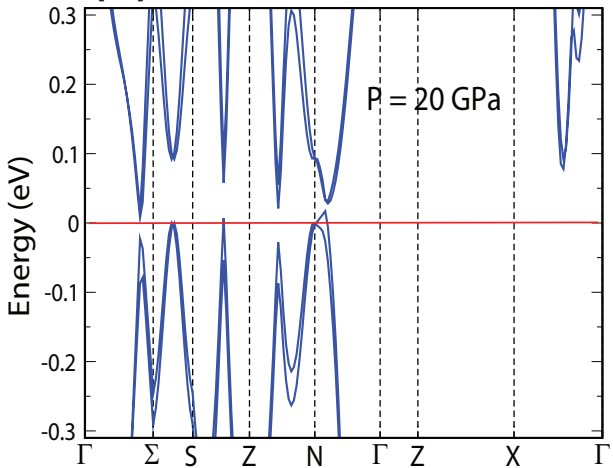

(d)

Figure 10. (Color online) - Electronic structure of bulk NbAs calculated at (a) 0 GPa, (b) $12 \mathrm{GPa}$, (c) $16 \mathrm{GPa}$ and (d) $20 \mathrm{GPa}$.

of the hexagonal phase of TaAs at higher pressures, shown in Fig.6 inset. There is only one Raman active mode $\left(\mathrm{E}^{\prime}\right)$ in the hexagonal phase of TaAs. The calculated frequency of $\mathrm{E}^{\prime}$ mode is very close to the $\mathrm{A}_{1}$ mode of $\mathrm{BCT}$ structure after $\mathrm{P}_{c}^{T a}$ and therefore the mode $\mathrm{A}_{1}$ marked in Fig.6 (a) can be the $\mathrm{E}^{\prime}$ mode of hexagonal phase after $\mathrm{P}_{c}^{T a}$. In Fig.6 (a), the other modes of BCT phase continue to exist after $\mathrm{P}_{c}^{T a}$, implying that the phase BCT continues to coexist along with the h-TaAs.

\section{Summary}

The phonon frequencies of all the Raman modes of NbAs show a change in $\mathrm{S}$ at $\mathrm{P}_{c}^{N b} \sim$ 15GPa. The pressure dependent volume and resistivity of NbAs show that there is a change in the bulk modulus and minimum in the resistivity at $\mathrm{P}_{c}^{N b}$. These anomalies are assigned to the pressure induced Lifshitz transition at $\mathrm{P}_{c}^{N b}$ in NbAs using first-principles DFT calculations. The change in $\mathrm{S}$ of the phonon frequencies of the first order Raman modes of TaAs and the minimum observed in resistivity at $\mathrm{P}_{c}^{T a} \sim 16 \mathrm{GPa}$ were attributed to structural phase transition in TaAs based on first-principles DFT calculations and high pressure synchrotron x-ray diffraction results. The symmetry of Raman modes in high pressure hexagonal phase of TaAs is also identified along with its phonon dispersion. 


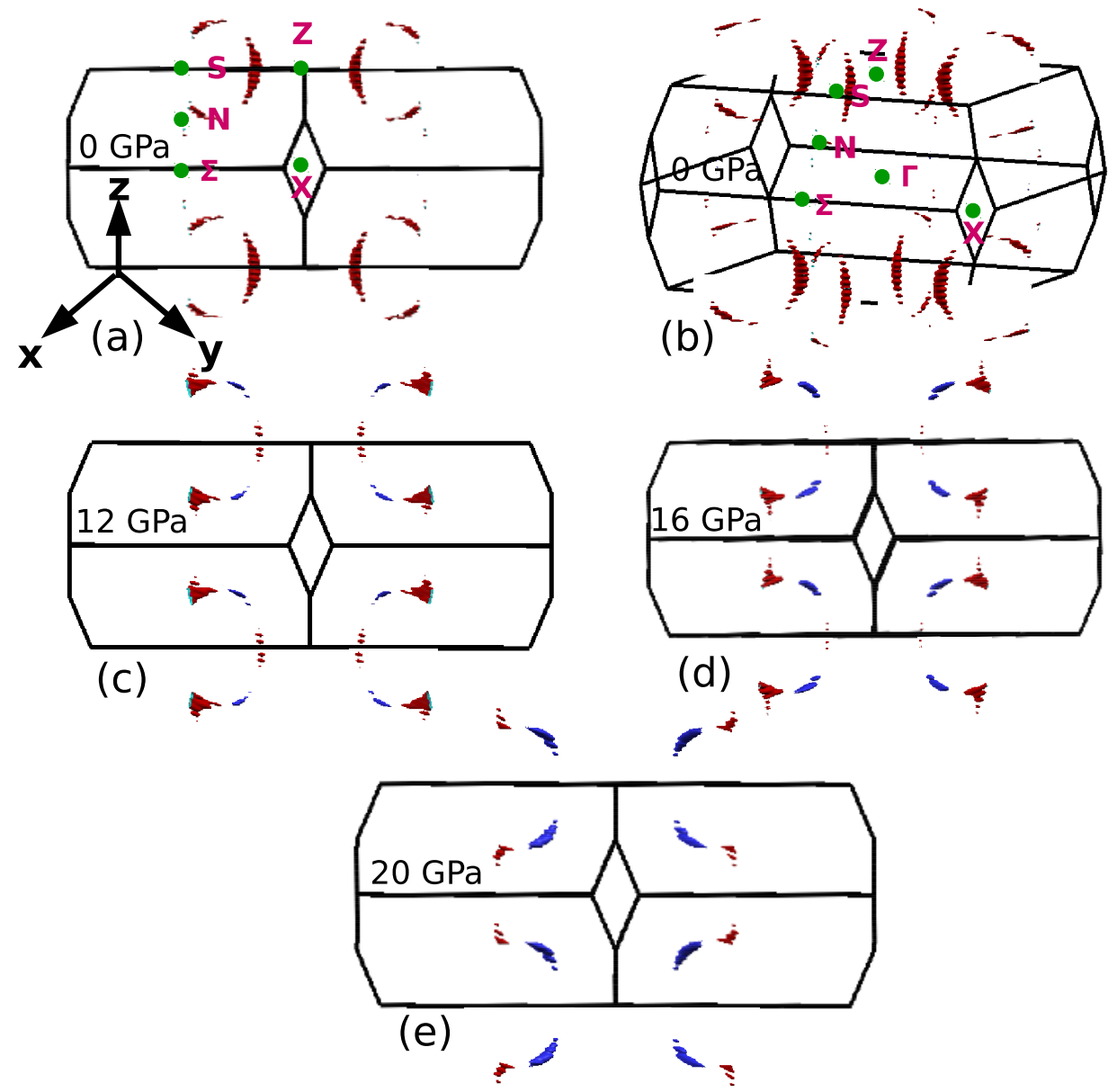

Figure 11. (Color online)-Evolution of the Fermi surface of NbAs with pressure. Two views of the Fermi surface at 0 GPa (a, b). Fermi surfaces at (c) 12 GPa (d) 16 $\mathrm{GPa}$ and (e) $20 \mathrm{GPa}$ respectively reveal changes in the size of electron and hole pockets with pressure. Red color shows hole pockets and blue color shows electron pockets.

\section{Acknowledgments}

We thank beam scientists Dr. Konstantin Glazyrin (P02, DESY) and Dr. Boby Joseph (Xpress, ELETTRA) for setting up the instruments to carry out the experiments. We gratefully acknowledge DESY, Germany and ELETTRA, Italy synchrotron XRD facility for high pressure x-ray experiments. We thank Department of Science and Technology (DST), India for financial support to carry out the experiments at DESY, Germany and Elettra, Italy. AKS thanks Department of Science and Technology (DST), India for the financial support. SNG thanks DST for the research fellowship. KP and AS thank JNCASR for research fellowships and acknowledge TUE-CMS for supercomputing facilities. UVW thanks DST support through a JC Bose National Fellowship.

\section{References}

[1] Weng H, Fang C, Fang Z, Bernevig B A and Dai X 2015 Phys. Rev. X 5(1) 011029

[2] Lv B, Weng H, Fu B, Wang X, Miao H, Ma J, Richard P, Huang X, Zhao L, Chen G et al. 2015 Physical Review X 5031013 

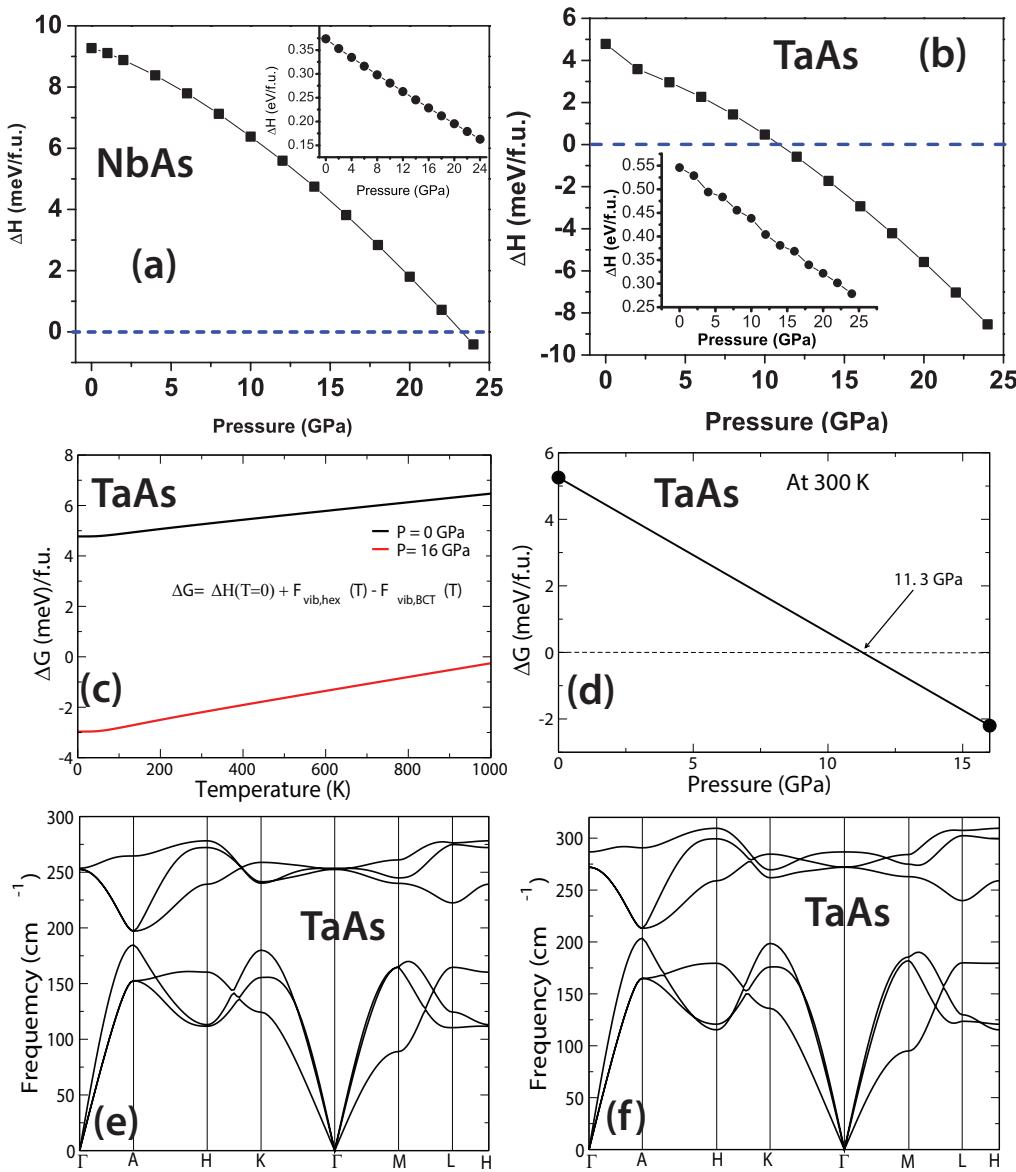

Figure 12. (Color online)-(a) Pressure dependent difference in enthalpies of NbAs in body-centered tetragonal ( $\left.\mathrm{I}_{1} \mathrm{md}\right)$ and hexagonal (P-6m2) structures, and body centered tetragonal and cubic (Pm-3m) structures (inset). (b) Enthalpy differences of the hexagonal and BCT structures of TaAs and enthalpy difference between the cubic and BCT structure of TaAs (inset). (c) Difference in Gibbs' free energy $(\Delta \mathrm{G})$ at 0 $\mathrm{GPa}$ (black line) and $16 \mathrm{GPa}$ (red line) as a function of temperature. (d) Interpolation of $\Delta \mathrm{G}$ predicts the transition pressure to be $11.3 \mathrm{GPa}$ at $300 \mathrm{~K} . \mathrm{F}_{v i b}$ is the vibrational contribution to the free energy. Phonon dispersion of the hexagonal structure of TaAs at (e) $0 \mathrm{GPa}$ and (f) $14 \mathrm{GPa}$ that do not exhibit any imaginary frequency. This implies the stability of the hexagonal crystal structure at ambient and higher pressures.

[3] Xu S Y, Belopolski I, Alidoust N, Neupane M, Bian G, Zhang C, Sankar R, Chang G, Yuan Z, Lee C C et al. 2015 Science $\mathbf{3 4 9} 613$

[4] Yang L, Liu Z, Sun Y, Peng H, Yang H, Zhang T, Zhou B, Zhang Y, Guo Y, Rahn M et al. 2015 Nature physics 11728

[5] Nielsen H B and Ninomiya M 1983 Physics Letters B 130389

[6] Aji V 2012 Physical Review B 85241101

[7] Son D and Spivak B 2013 Physical Review B 88104412

[8] Kim H J, Kim K S, Wang J F, Sasaki M, Satoh N, Ohnishi A, Kitaura M, Yang M and Li L 2013 Physical review letters 111246603

[9] Hosur P and Qi X 2013 Comptes Rendus Physique 14857

[10] Huang X, Zhao L, Long Y, Wang P, Chen D, Yang Z, Liang H, Xue M, Weng H, Fang Z et al. 2015 Physical Review X 5031023 
[11] Zhang C L, Xu S Y, Belopolski I, Yuan Z, Lin Z, Tong B, Bian G, Alidoust N, Lee C C, Huang S M et al. 2016 Nature communications 710735

[12] Shekhar C, Nayak A K, Sun Y, Schmidt M, Nicklas M, Leermakers I, Zeitler U, Skourski Y, Wosnitza J, Liu Z et al. 2015 Nature Physics 11645

[13] Huang S M, Xu S Y, Belopolski I, Lee C C, Chang G, Wang B, Alidoust N, Bian G, Neupane M, Zhang C et al. 2015 Nature communications 67373

[14] Lv B, Xu N, Weng H, Ma J, Richard P, Huang X, Zhao L, Chen G, Matt C, Bisti F et al. 2015 Nature Physics 11724

[15] Xu S Y, Belopolski I, Sanchez D S, Zhang C, Chang G, Guo C, Bian G, Yuan Z, Lu H, Chang $\mathrm{T} \mathrm{R}$ et al. 2015 Science advances 1 e1501092

[16] Xu N, Weng H, Lv B, Matt C, Park J, Bisti F, Strocov V, Gawryluk D, Pomjakushina E, Conder $\mathrm{K}$ et al. 2016 Nature communications 711006

[17] Zhou Y, Lu P, Du Y, Zhu X, Zhang G, Zhang R, Shao D, Chen X, Wang X, Tian M et al. 2016 Physical Review Letters 117146402

[18] Luo Y, Ghimire N, Bauer E, Thompson J and Ronning F 2016 Journal of Physics: Condensed Matter 28055502

[19] dos Reis R, Wu S, Sun Y, Ajeesh M, Shekhar C, Schmidt M, Felser C, Yan B and Nicklas M 2016 Physical Review B 93205102

[20] Shekhar C, Süss V and Schmidt M 2016 arXiv preprint arXiv:1606.06649

[21] Giannozzi P, Baroni S, Bonini N, Calandra M, Car R, Cavazzoni C, Ceresoli D, Chiarotti G L, Cococcioni M, Dabo I et al. 2009 Journal of Physics: Condensed Matter 21395502

[22] Dal Corso A 2014 Computational Materials Science 95337

[23] Perdew J P and Zunger A 1981 Phys. Rev. B 23(10) 5048

[24] Baroni S, De Gironcoli S, Dal Corso A and Giannozzi P 2001 Reviews of Modern Physics 73515

[25] Ghimire N J, Luo Y, Neupane M, Williams D, Bauer E and Ronning F 2015 Journal of Physics: Condensed Matter $\mathbf{2 7} 152201$

[26] Liu H, Richard P, Zhao L, Chen G and Ding H 2016 Journal of Physics: Condensed Matter 28 295401

[27] Einaga M, Shimizu K, Hu J, Mao Z and Politano A 2017 physica status solidi (RRL)-Rapid Research Letters 111700182

[28] Petříček V, Dušek M and Palatinus L 2014 Zeitschrift für Kristallographie-Crystalline Materials 229345

[29] Xiao W, Tan D, Xiong X, Liu J and Xu J 2010 Proceedings of the National Academy of Sciences 10714026

[30] Zhao J, Xu L, Liu Y, Yu Z, Li C, Wang Y and Liu Z 2015 The Journal of Physical Chemistry C 11927657

[31] Hong F, Yue B, Hirao N, Ren G, Chen B and Mao H K 2016 Applied Physics Letters 109241904

[32] Belsky A, Hellenbrandt M, Karen V L and Luksch P 2002 Acta Crystallographica Section B: Structural Science $\mathbf{5 8} 364$

[33] Lee C C, Xu S Y, Huang S M, Sanchez D S, Belopolski I, Chang G, Bian G, Alidoust N, Zheng H, Neupane M et al. 2015 Physical Review B 92235104

[34] Bera A, Singh A, Muthu D, Waghmare U and Sood A 2017 Journal of Physics: Condensed Matter 29105403 